\title{
Scaphoid Fractures Treated With Kirschner (K) Wires or Herbert Screw Fixation Has No Difference in Fracture Healing Period
}

\author{
Authors \\ Ashutosh Kumar', Manish Ranjan², Mukesh Kumar ${ }^{3}$, Neeraj Kumar \\ Ravi Kumar ${ }^{5}$, Chandan Kumr ${ }^{6}$, Amarjeet Kumar ${ }^{7}$ \\ 1,3,4,5,6, Senior Resident, Orthopedics Dept, AIIMS, Patna \\ ${ }^{2}$ Assistant Professor, Orthopedics Dept, PMCH, Patna \\ ${ }^{7}$ Senior Resident, Anesthesia Dept, AIIMS, Patna
}

\begin{abstract}
Background: The scaphoid is the most common fractured carpal bone. These fractures are commonly difficult to diagnose or go undetected. Degenerative changes set in if scaphoid fracture non-union is left untreated. This study evaluates the outcome of scaphoid fracture treated with $K$ wires or Herbert screw and bone grafting. Materials and Methods: 7 cases of scaphoid fracture treated from november 2014 to june 2016 were treated with $K$ wires or Herbert screw and bone grafting. Scaphoid nonunion was both clinically and radio graphically diagnosed. Fractures were graded according to herbert's classification.

Results: 7 patients could be followed up. All patients were no intra operative complications. One (1) patient developed post-operative infection. The study contained 5 male and 2 female subjects. The average age was 29.6 years. Dominant wrist was affected in 5 patients, and non-dominant was affected in 2. Most of the patients showed evidence of radiological union between 14 to 16 weeks. Range from 12 to 23 weeks and an average of 16.40 weeks. Most of the patients 19 (67\%) recovered $80 \%$ or more of grip strength.

Conclusion: Our findings suggest that 1) Scaphoid fracture treated with Kirschner-wire or Herbert screw fixation and iliac crest bone grafting presents a 90\% healing rate at 6 months follow up. 2) Scaphoid fracture treatment with Kirschner-wire or Herbert screw fixation and iliac crest bone grafting significantly decreases pain and achieves 84\% wrist joint motion compared to opposite side. 3) Scaphoid fracture treatment Kirschner-wire fixation or Herbert screw and iliac crest bone grafting yields $75 \%$ of satisfactorily functional results. 4) Average time for radiological union is $16.40 \%$ weeks.

Keywords: Scaphoid fracture, Kirschner-wire or Herbert screw fixation, iliac crest bone grafting.
\end{abstract}

\section{Introduction}

The scaphoid is the most common fractured carpal bone. ${ }^{[1-3]}$. These fractures cannot diagnose easily or go undetected ${ }^{[4]}$. If the patient does not take medical attention upon considering that it was a trivial wrist trauma, or if the patient is not treated by immobilized for adequate period of time, fracture healing will not occur, because of atypical vascularity of scaphoid bone ${ }^{[5]}$. The mostly scaphoid fracture treatment is controversial. The aim of treatment is bone healing with scaphoid shape restoration and ligaments preservation. Some cases show mild symptomatic with mild functional deficit. But most cases, has tendency for progressive arthrosis. They may be treated by electric or electromagnetic stimulation ${ }^{[6]}$, bone grafting ${ }^{[7]}$, internal fixation ${ }^{[8]}$, vascularized bone grafting ${ }^{[9,10]}$, and combined methods ${ }^{[11]}$. Fracture 
healing failure rate with non-union persistence is also high, with prolonged immobilization [7], prevent patients to perform their professional work, not only during rehabilitation period, but also during postoperative rehabilitation, leads to serious socioeconomic problem ${ }^{[2]}$. Degenerative changes occur if scaphoid fracture non-union is left untreated. An asymptomatic patient with an undisplaced non-union fracture with no evidence of the wrist will also develop late degenerative changes at their carpal bone ${ }^{[19]}$. This study focus on the result of scaphoid fracture treated with $\mathrm{K}$ wires or Herbert screw fixtation and bone grafting.

\section{Methods and Materials}

This study was carried out at Aiims, Patna during november 2014 to june 2016. The subjects were patients who were established cases of displaced scaphoid fracture. Patients who had taken primary treatment elsewhere were also included.

\section{Inclusion Criteria}

$>$ Established cases of displaced scaphoid fracture.

$>$ Clinically pain and decreased ROM at wrist.

$>$ Radiological signs of displaced scaphoid fracture, nonunion in terms of sclerosis, cystic changes or a vascularity of proximal fragment.

\section{Exclusion criteria}

$>$ Other associated fractures, dislocations or ligament injuries of the same wrist.

Arthritis of the affected wrist.

\section{Methods}

Only cases of established displaced fracture treated during November 2014 to June 2016 with iliac crest bone grafting and $\mathrm{K}$ wire or Herbert screw fixation were selected. Dominance Hand, mechanism of injury, interval of injury and treatment, anatomical location of fracture was noted. Profession of the patients abd inability to perform manual work was noted. Patient other complains like was noted. Scaphoid fracture with non-union was both clinically and radiographically diagnosed. Complain of patients were wrist pain, decreased strength and inability to perform any manual work. On physical examination, pain was reproduced during direct compression of the scaphoid or axial loading of the thumb. On plain x-ray, with anterior-posterior, lateral and oblique wrist views as well as clenched fist views, taken for scaphoid non-union was demonstrated. Nonunion scaphoid may show smooth round fracture edges, osteosclerosis and bone cyst formation. Fractures were classified according to Herberts classification. Patients were treated with iliac crest bone grafting with open $\mathrm{K}$ wire or Herbert screw fixation. Post-operatively the wrist was immobilized with plaster involving the metacarpo-phalangeal joint of the thumb for a period of three months or till evidence of radiological union was seen. All 7 patients could be followed up. Patients were interviewed and examined. Preoperatively patients were examined for range of motion using a goniometer compared to opposite hand. Pre op and post op $\mathrm{X}$ rays (immediate, 1 month and 3 months or till fracture united) were taken.

Post operative analysis was done: Post operatively patient was assessed by modified scaphoid scoring system for pain, range of motion at wrist, wrist strength, return to work and union of non union site. Grip strength was compared to opposite hand and was graded as percentage as compared to opposite hand.

\section{Technique}

After proper antisepsis condition and after the pneumatic tourniquet was installed. The upper limb was elevated for 5 minutes and then exsanguinated. The pneumatic tourniquet was then elevated to $130 \mathrm{~mm} \mathrm{Hg}$ pressure.

A 4-5 $\mathrm{cm}$ incision was carried out from the radial edge of the flexor carpi radialis over the tip of the styloid process of the radius. Flexor carpi radialis tendon and the radial artery were isolated. The palmar joint capsule was longitudinally divided 
and the deep palmar radio-carpal ligaments (radioscapho-capitae and radioluanate) were also divided and repaired at the end of the operation or mobilized. The fracture site were exposed- the proximal and distal fragments, and the scapholunate junction. The fibrous and sclerotic tissue were removed with the use of a small currate until fresh hemorrhagic cancellous bone was revealed. After the making of the scaphoid cavity, cortical bone was harvested from the iliac crest or distal radius of same wrist to restore the scaphoid height and cancellous bone was taken to fill the cavity. The cortical bone graft was then inserted into the scaphoid cavity in a manner that two scaphoid fragments get appropriate distracted and correcting the scaphoid height. After insertion of the graft, the protruding edges were maintain with the proximal and distal fragments. Osteosynthesis now performed with the use of two Kirschner wires or Herbert screw which were inserted in parallel with the longitudinal axis of the scaphoid were selected. The alignment of scaphoid and position of $\mathrm{K}$ wires or Herbert screw fixation was checked in anterio-posterior and oblique views under image intensifier. The radiocarpal ligaments and the joint capsule were then repaired with vicryl. The wrist was immobilized in a palmar slab for 2 weeks. The sutures were removed after 2 weeks, and the long arm thumb spica cast was applied for 1 month. All patients

\section{Fig:1}

were instructed to followed a specific physical therapy protocol immediately after the operation. The physical therapy included finger, elbow and shoulder exercise during the immobilization period. The cast was changed every month and $\mathrm{X}$ rays done. If healing was satisfactory casting was continued for additional few weeks. Most patients had wires removed at the same time as the cast removed. The $\mathrm{K}$ wires were removed without any anesthesia. After the clinical and radiological confirmation of bone healing (14.8 weeks) was done, the cast was removed and the patients were instructed to perform active, passive and assisted active movement of the wrist and forearm. After regaining satisfactory range of movement the patients followed a mild vegirous protocol. All patients were allowed to return to heavy manual work and to sports after a period of six months or three months after the healing. All patients were assessed by using Modified Scaphoid outcome Scoring System ${ }^{[13]}$. The scoring system consisted of scores for pain, wrist strength, ROM at wrist, return to work and patient satisfaction. Also the union of non-union site was assessed by $\mathrm{x}$ ray and time period documented. Grip strength was recorded in operated hand with percentage of grip the opposite hand. Tabulating this data, various variables were statistically examined for their importance and relevance in functional outcome and radiological union

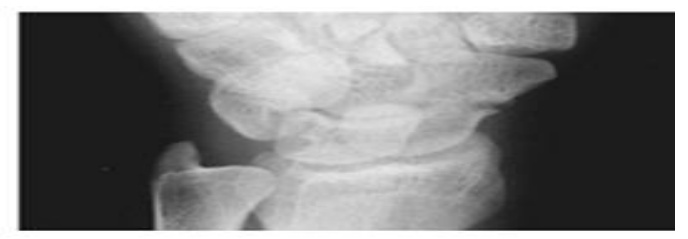

Pre op $\times$ rays
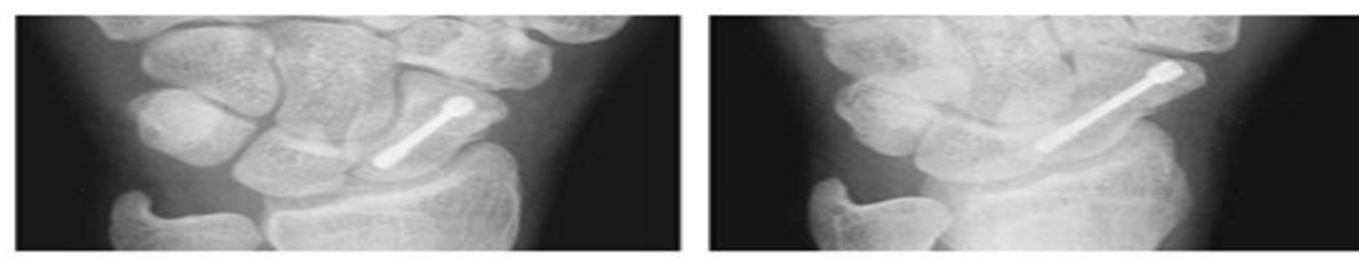

post operative $x$ rays Herbert screw fixation 
Fig:2
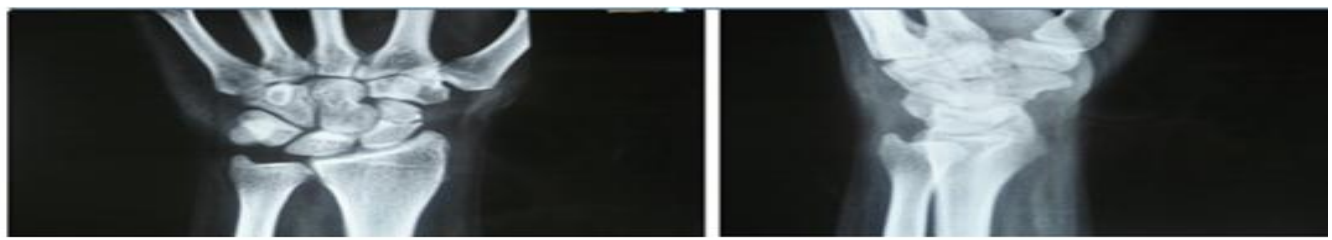

Pre op $\times$ rays
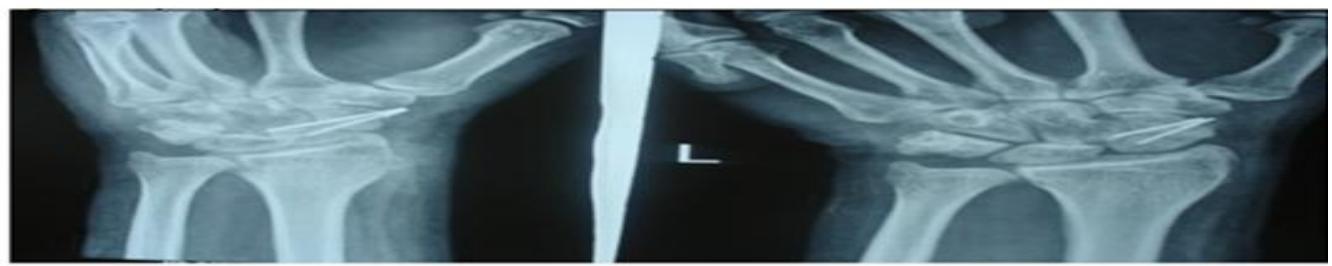

post operative $X$ rays with $K$ wires fixation

\section{Results}

All 7 patients were operated between november 2014 to june 2016. There were no intra operative complications. Post operatively 1 patient developed mild infection but healed with proper dressing and short course of antibiotics. This study contained 5 male and 2 female subjects. The ages of the patients in the study ranged from 21 to 48 years. The average age was 29.6 years. $75 \%$ of the patients were younger than 35 years of age. Dominant wrist was affected in 5 patients, and non-dominant was affected in 2.

The range of motion (Flexion, Extension, Radial and Ulnar deviation) of the operated wrist was compared to the opposite side. The mean loss of flexion extension was about $14 \%$ compared to opposite side and mean loss in radial-ulnar deviation was $19 \%$. Most of the patients showed evidence of radiological union between 14 to 16 weeks. Range from 12 to 23 weeks and an average of 16.40 weeks. Most of the patients returned back to there original profession. Most of the patients had little or no pain. 2 out of the 7 patients had pain on exertion of the wrist and took occasional analgesics. 1 patient who had poor result complained of persistent pain. Grip strength was subjectively assessed and compared to opposite hand by the same examiner to minimise bias. Most of the patients has good grip strength. Mean grip strength at follow up was $80 \%$ compared to opposite hand.

\section{Discussion}

Mechanism of injury did not seem to affect the result. One of the patients who failed to unite after the operation had sustained injury as a result of direct blow. We left the plaster on for about 2 weeks after we confirmed that the fracture has united radiographically. Mean time for immobilization in plaster was 16.25 weeks in the study. Stark et al. ${ }^{[12]}$ kept the plaster on for 17 weeks in their study which shows $90 \%$ positive outcome.

One of the probable advantages of screw fixation over the k-wires is that it allows early mobilization. A meta analysis ${ }^{[18]}$ found the average union rate to be same in four studies that used no immobilization and other studies shows that used more than 6 weeks of immobilization. 4 to 12 weeks of immobilization are often reported for non-union of the scaphoid ${ }^{[14,16]}$. It has been seen that screw over $\mathrm{K}$ wire fixation the long time in plaster did not seem to adversely affect the range of motion in our patients. The mean loss of flexion-extension was about $14 \%$ compared to opposite side and mean loss in radial-ulnar deviation was $19 \%$. This is similar to or better than the mobility reported after screw fixation in spite of shorter immobilization ${ }^{(14,15,16)}$. The time in plaster of Paris for our patients is not quite as long as the 4-6 months recommended after Russe operation ${ }^{[17]}$. Only 2 of the 5 patients had poor result. The success rate was in the region was high 
confirming the good results reported by Stark et $\mathrm{al}^{[12]}$.

In a recent meta-analysis of 12 studies comprising of 519 patients treated with bone graft and screw the union rate was $74 \%$. Herbert screws are less likely to impinge on adjacent bones than lag screws, but have been reported to be difficult to position correctly ${ }^{[13]}$. Trumble et al. ${ }^{[14]}$ reported that only 7 of their 16 Herbert screws were ideally placed. Inoue et al. ${ }^{[15]}$ reported that 3 of their 16 Herbert screws were misplaced and according to Rajagopalan et al. ${ }^{[6]}$ out of the 21 Herbert screws 1 migrated into the joint and 2 loosened. We had no intra operative problems in our series. Most our patients had no or little pain. None of the patients had to take long-term pain killer. Time away from work was longer than 11 weeks reported by Herbert screw fixation ${ }^{[15]}$. Most of the patients returned back to their previous profession in 17 weeks. Scaphoid outcome scores were comparable those reported after Herbert screw fixation and kwire fixation ${ }^{[14]}$. The average grip strength in operated hands was $80 \%$ compared to the opposite hand. Grip strengths of 80 and $90 \%$ have been reported after bone grafting and Herbert screw fixation ${ }^{[15,16]}$. Scaphoid outcome score were not as good as those reported after screw fixation. In this study 5 patients had excellent to good scores while Rajgopalan et al. found this in 18/21 unselected pseudoarthroses and Robbins et al. in 11/17 proximal pole nonunion. The much longer follow up in our patients may be of significance. However, in spite of the long follow up none of the patients had developed arthritic changes.

\section{Conclusion}

1. Scaphoid fracture treated with Kirschnerwire or Herbert screws fixation and iliac crest bone grafting presents a $90 \%$ healing rate at 6 months follow up.

2. Scaphoid fracture treated with Kirschnerwire or Herbert screws fixation and iliac crest bone grafting significantly decreases pain and achieves $85 \%$ wrist joint motion compared to opposite side.
3. Scaphoid fracture treated with Kirschnerwire or Herbert screws fixation and iliac crest bone grafting yields $75 \%$ of satisfactorily functional results.

4. Average time for radiological union is $16.40 \%$ weeks.

5. Scaphoid fractures treated with kirschner (k) wires or herbert screw fixation has no diffrence in fracture healing peroid

\section{References}

1. Van der Molen A.B., Groothoff J.W., Visser G.J.P., Robinson P.H., Eisma W.H.:Time off work due to scaphoid fractures and other carpal injuries in the Netherlands in the period 1990 to 1993 . J Hand Surg [Br] 1999; 24: 193-198.

2. Shuind F., Haentjens P., van Innis F., van der Marren C., Garcia-Elias M., Sennwald G.: Prognostic factors in the treatment of carpal scaphoid nonunions. J Hand Surg [Am] 1999;24: 761-76.

3. Watson H.K., Pitts E.C., Ashmead D. 4th, Makhlouf V.M., Kauer J.: Dorsal approach to scaphoid nonunion. J Hand Surg [Am] 1993; 18: 359-65.

4. Berná J.D., Abaledejo F., Sanchez Cañizares M.A., Chavarria G., Pardo A., Pellicer A.: Scaphoid fractures and nonunions: a comparison between panoramicradiography and plain rays. J Hand Surg [Br] 1998; 23: 328-31.

5. Taleisnik J., Kelly P.J.: The extraosseous and intraosseous blood supply of the scaphoid bone. J Bone Joint Surg [Am] 1966; 48: 1125-37.

6. Adams B.D., Frykman G.K., Taleisnik J.: Treatment of scaphoid nonunion with casting and pulsed electromagnetic fields: a study continuation. J Hand Surg [Am], 1992; 17: 910-4.

7. Russe O.: Fracture of the carpal navicular. J Bone Joint Surg [Am] , 1960; 42: 75968. 
8. Huene D.R., Huene D.S.: Treatment of nonunions of the scaphoid with the ender compression blade plate system. J Bone Joint Surg [Am] , 1991; 16: 913-922.

9. Zaidemberg C., Siebert J.W., Angrigiani C.: A new vascularized bone graft for scaphoid nonunion. J Hand Surg [Am] , 1991; 16: 474-8.

10. Yuceturk A., Isiklar Z.U., Tuncay C., Tandogan R.: Treatment of scaphoid nonunions with a vascularized bone graft based on the first dorsal metacarpal artery. J Hand Surg [Br] 1997; 22: 425-7.

11. Kuhlmann J.N., Minoun M., Boabighi A., Baux S.: Vascularized bone graft pedicled on the volar carpal artery for non-union of the scaphoid. J Hand Surg [Br] 1987; 12: 203-10.

12. Stark HH, Richard TA, Zemel NP, Ashworth CR. Treatment of ununited fractures of the scaphoid by iliac crestbone graft and Kirschner wire fixation.JBJS 1988; 70-A: 98291.

13. Robbins RR, Ridge O, Carter PR. Iliac crest bone grafting and Herbert screw fixation of nonunions of scaphoid with avascular proximal poles. J Hand Surgery1995; 20-A: 818-31

14. Trumble T. E. , Clark T. , Kreder H. J. Nonunion of the Scaphoid. Treatment with cannulated screws compared with treatment with Herbert Screw. J.B.J.S. 1996:78A:1829-3.

15. Inoue G., Shionoya K., Yoshio K., Ununited proximal pole fractures. Treatment with Herberts Screw in 16 cases followed for .5 to 8 years. Acta Orthop. Scand. 1997.

16. Rajagopalan B. M., Squire D. S., Samuels L. O., results of Herbets screw fixation with bone grafting for the treatment of non-union of the scaphoid. J.B.J.S. 1999:81A: 48-52.

17. Green D. P. the effect of avascular necrosis on Russe bone grafting for nonunion scaphoid. J Hand Surg.1985; 10A: 597-605.

18. Merrell G.A., Wolfe S.W., Slade J.F., treatment of scaphoid non-union : quantitative meta-analysis of the literature. J Hand Surg 2002; 27A:685-91.

19. Commander Gregory R, et al, The natural History of Scaphoid nonunion, J.B.J.S 1984; 66-A(4):.504-7. 